\title{
Aus dem Vorworte zur zweiten Auflage.
}

Ebenso wie der erste ist auch dieser zweite Band einer gründlichen Durchsicht unterzogen worden; doch litg auch hier kein AnlaB vor, den Gang der Entwicklungen wesentlich abzuändern, abgesehen von einer Stelle:

Die Bestimmung der Flächen mit gegebenen und den drei Fundamentalgleichungen Genüge leistenden FundamentalgröBen konnte ich diesmal, von einer Bemerkurg ExGELs Gebrauch machend, derirt in mehrere Teile zerlegen, daB die schwierigkeiten für den Anfänger recht reningert werden: In $\S 3$ des dritten Abschnittes wird zunächst nur das Vorbındensein einer Fläche mit den gegebenen Fund:ımentalgrößen bewiesen; mittels der in $\$ 7$ aufgestellten Differenti:alinvarianten wird alsdann gezeigt, daßB alle Flächen mit denselben Fundament:ılgrößen ein:ınder kongruent sind. Mit diesen beiden Ergebnissen kann man sich, wenn man will, begnügen. Nachträglich wird jedoch in den tubcrschlagbaren $\$ \S 8$ und 9 des dritten Abschnittes erörtert, wie man eine unter diesen Flächen und damit auch alle durch Integration einer Riccatischen Gleichung und einige Quildraturen zu bestimmen vermag.

Fine andere mehr untergeordnete Abänderung ist diese: In der ersten Auflige bitte ich die natürlichen Gleichungen einer Fläche, die Hauptkrümmungsradien zuläBt, d:durch zum Ausdrucke gebracht, daB . ich die Ableitungen dieser Radien längs der Krümmungskurven als Funktionen der Radien ditrstellte. Die hierbei auftretenden GröBen sind nun aber keine absoluten Invari:nnten gegenüher Bewegungen und gegenüber der Einführung neuer Parameter. Deshalb habe ich es diesmal vorgezogen, statt der Radien das Krümmungsm: $B \quad K$ und das Quadrat der mittleren Krümmung $H$ zu benutzen, die ja absolute Invarianten sind. Aus ihnen werden mit Hilfe der Differentialparameter vier andere absolute Invariınten gebildet, und nun sind die natürlichen Gleichungen der Fläche diejenigen Gleichungen, die aussingen, wie sich diese letzten vier Invarianten als Funktionen von $K$ und $I^{2}$ darstellen. Selbstrerständlich kann man von diesen natürlichen Gleichungen wieder zu denen zurückgelangen, die in der ersten Auflage benutzt wurden (vgl. S. 443). Die Flächen, auf denen $K$ und $H$ voneinander abhängig sind, oder wie man nicht ganz einwandfrei zu sagen plegt, eine Gleichung $z$ wischen den beiden Hauptkrummungsradien besteht, sind bei jenen Betrachtungen auszuschlieBen. Auf die Frage, wie man für sie zweckmüBig die natürlichen Gleichungen aufstellt, bin ich nicht näher eingegangen. 
Im ersten Abschnitte ist der $\S 4$ über die Einhullende einer zweifach unendlichen Ebenenschar neu.

Der zweite Abschnitt ist um folgende Paragraphen bereichert worden: $\S 5$ über die Schnittkurve von Fläche und Tangentenebene, \$ 7 über oskulierende Flächen zweiter Ordnung, § 12 über dreifache orthogonale Flächensysteme, § 17 über Flächen von Minimalgeraden. Viele andere geringfügigere Zusätze finden sich an den verschiedensten Stellen des Buches. Die wie im ersten Bande in der neuen Auflage auch hier überall durchgeführte naturgemäBe Orientierung des räumlichen Koordinatensystems erforderte die Umänderung mehrerer Figuren, außerdem wurden mehrere neue $\mathrm{Ab}$ bildungen eingeschaltet. Insbesondere möchte ich auf die Fig. 93, S. 363, hinweisen, die die stetige Verbiegung einer gemeinen Schraubenfläche auf ein Katenoid veranschaulicht.

Berlin-Steglitz, im August 1913.

\section{Vorwort zur dritten Auflage.}

Diesmal habe ich mich in der Hauptsache darauf beschränkt, die mir bekannt gewordenen Druckfehler und Versehen auszubessern. AuBerdem sind nur einige durchgreifendere Änderungen vorgenommen worden: Auf Grund ron wertrollen Rutschiägen meines Freundes ENGEI, konnte ich einige Betrachtungen wesentlich vereinfachen, nämlich den \& 17 des zweiten Abschnittes uber die Flächen von Minimalgeraden und den $\$ 8$ des dritten Abschnittes über den Ansatz zur Ermittelung einer Fläche mit gegebenen FundamentalgröBen, im Zusammenhange hiermit auch den auf den Fall $k=G=0$ bezüglichen Teil des \& 9 desselben Abschnittes.

Selbstrerständlich hätte ich gern das ganze Buch einer durchgreifenden Erneuerung unterzogen; aber wegen der gewaltig gestiegenen Kosten für die Herstellung von Büchern war es geboten, den alten Text möglichst schonend zu behandeln, damit das Buch nicht gar zu teuer werde. Deshalb habe ich auch auf die Erfullung einiger anderer Wunsche von befreundeter Seite verzichten mussen. Ich hoffe, daB das Buch trotz alledem seinem Zwecke, dem Anfänger zu einer wirklich auch geometrischen Auffassung der Flächentheorie zu verhelfen, wio bisher so auch in Zukunft dienen wird.

Berlin-Dahlem, im April 1922. 